in understanding and addressing the needs of women with OF: clinical care, academia, international health, civil society, and government. Twenty-one individuals were interviewed about their perceptions of IDs for OF self-management and their implementation. Interviews were audio-recorded and transcribed. The Consolidated Framework for Implementation Research (CFIR) guided data collection and analysis. Thematic analyses were carried out within Nvivo v.12. RESULTS/ANTICIPATED RESULTS: Determinants of implementation of an ID for OF self-management (by CFIR domain) include: (1) intervention characteristics-relative advantage and cost; (2) individual characteristics—knowledge and beliefs about the innovation; (3) inner setting-organizational culture, implementation climate, tension for change, and compatibility; (4) outer setting-patient needs and resources and external policy and incentives; (5) process-opinion leaders and collaboration. Facilitators include: tension for change for low-cost, accessible IDs; relative advantage over existing tools; development of partnerships; and identification of implementation champions. Barriers include: need for educational strategies to encourage clinical provider acceptability; lack of evidence of the optimal beneficiary. DISCUSSION/SIGNIFICANCE OF IMPACT: Tools for therapeutic OF self-management could be integrated into comprehensive OF programming. Employing the CFIR as an overarching typology allows for comparison across contexts and settings where OF care occurs and may be useful for clinicians, researchers, and policy-makers interested in implementing IDs for OF self-management in LMICs. CONFLICT OF INTEREST DESCRIPTION: I am working with colleagues at the non-profit Restore Health on developing an insertable cup for therapeutic self-management of obstetric fistula in LMICs

4432

Transportation Barriers and Preferences Among Drivers with Developmental Disabilities in Southeast

Austin Svancara ${ }^{1}$, Rajesh Kana ${ }^{2}$, Benjamin McManus ${ }^{1}$, Haley

Bednarz $^{1}$, Gabriela Sherrod ${ }^{1}$, and Despina Stavrinos ${ }^{1}$

${ }^{1}$ University of Alabama at Birmingham; ${ }^{2}$ University of Alabama

OBJECTIVES/GOALS: Transportation may be a barrier for individuals with Autism Spectrum Disorder (ASD). More individuals with ASD utilize public transportation compared to typically developing (TD) individuals. This study seeks to elucidate the transportation needs of individuals with ASD in the Southeast. METHODS/ STUDY POPULATION: Sixty-one licensed drivers with a diagnosis of ASD ( $\mathrm{n}=21)$, Attention-Deficit/Hyperactivity Disorder (ADHD; $\mathrm{n}=19$ ), or no diagnosis (TD; $\mathrm{n}=21$ ) were recruited and were matched across diagnosis groups by age (16-30 years old), gender, and IQ. Participants completed an adapted version of the Barriers to Care Scale and a survey assessing transportation preferences and quality of life. Means and frequencies were obtained. Chi-square analyses were conducted to estimate associations between diagnosis and transportation preferences. RESULTS/ANTICIPATED RESULTS: Nearly all of the sample had access to a car (98.4\%). Yet, only $71.4 \%$ of drivers ASD preferred to use their own car compared to $89.5 \%$ and $90.5 \%$ of the ADHD and TD groups respectively. The use of public transportation (6.6\%) and ride-hailing services (18\%) for general transportation needs was very low across the groups. There was a significant association between group type and the reliance on others for transportation $\left(\chi^{2}(2,61)=9.9, p<\right.$ .01 ). Only $21.1 \%$ of those with ADHD relying on others for transportation needs, compared to $61.9 \%$ of TD and $66.7 \%$ of individuals with ASD. 23.8\% of ASD drivers, 10.5\% of ADHD drivers, and 9.5\% of TD drivers believe transportation proved as an obstacle. DISCUSSION/SIGNIFICANCE OF IMPACT: The proportion of ASD drivers who believed transportation to be a barrier appeared slightly higher than other groups. Public transportation use may be low due to lower accessibility to such services in the Southeast. The travel patterns of individuals with ASD and ADHD merits further exploration.

4345

\section{Two-step Algorithm for Clostridioides difficile is Inadequate for Differentiating Infection from Colonization in Children}

Maribeth R Nicholson ${ }^{1}$, Jacob M Parnell, Irtiqa Fazili, Sarah C. Bloch, D. Borden Lacy, Eric Skaar, and Kathryn M Edwards

${ }^{1}$ Vanderbilt University Medical Center

OBJECTIVES/GOALS: In 2017, new guidelines recommended multi-step algorithms for CDI diagnosis, and clinical centers rapidly implemented changes despite limited pediatric data. We assessed a multi-step algorithm using NAAT followed by EIA for ability to differentiate symptomatic CDI from colonization in children. METHODS/STUDY POPULATION: We prospectively enrolled pediatric patients with cancer, cystic fibrosis, or inflammatory bowel disease who were not being tested or treated for CDI and obtained a stool sample for NAAT. If positive by NAAT (colonized), EIA was performed. Children with symptomatic CDI who tested positive by NAAT via the clinical laboratory were also enrolled and EIA performed on residual stool. A functional cell cytotoxicity neutralization assay (CCNA) was performed in addition. RESULTS/ ANTICIPATED RESULTS: Of the 138 asymptomatic children enrolled, 24 (17\%) were colonized. An additional 37 children with symptomatic CDI were enrolled. Neither EIA positivity ( $41 \%$ versus $21 \%, P=0.11$ ) or CCNA positivity ( $49 \%$ versus $46 \%, P=0.84$ ) were significantly different between symptomatic versus colonized children. When both EIA and CCNA were positive, children were more commonly symptomatic than colonized ( $33 \%$ versus $13 \%, P=0.04$ ). DISCUSSION/SIGNIFICANCE OF IMPACT: A multi-step testing algorithm with NAAT and EIA failed to differentiate symptomatic CDI from colonization in our pediatric cohort. As multi-step algorithms are moved into clinical care, pediatric providers will need to be aware of the continued limitations in diagnostic testing.

\section{1}

Understanding barriers and solutions towards access to mental health among rural adolescents

Brandy Davis ${ }^{1}$, Kimberly B. Garza², Salisa Westrick², Edward Chou ${ }^{2}$, and Cherry Jackson ${ }^{2}$

${ }^{1}$ University of Alabama at Birmingham; ${ }^{2}$ Auburn University

OBJECTIVES/GOALS: There are two objectives: 1) To identify healthcare providers' (HCP) barriers and potential solutions towards 